Diabetologe 2016 $\cdot 12: 302-311$

DOI 10.1007/s11428-016-0111-8

Online publiziert: 30. Juni 2016

๑) Springer-Verlag Berlin Heidelberg 2016

CrossMark

\section{W. A. Scherbaum}

Universitätsklinikum Düsseldorf, Heinrich-Heine-Universität Düsseldorf, Düsseldorf, Deutschland

\title{
Diabetes Update 2016
}

\section{Hot Topics und Highlights, die der diabetologisch tätige Arzt kennen sollte}

Was im März 2016 beim traditionellen Diabetes Update in Mainz präsentiert und diskutiert wurde, ist mehr als eine Bestandsaufnahme der aktuellen Erkenntnisse in der Diabetologie. Es ist auch als kritische Auseinandersetzung mit unseren bisherigen diagnostischen und therapeutischen Prozeduren anzusehen. Ein Teil dieser neuen wissenschaftlichen Erkenntnisse wird sehr kurzfristig in die Diagnose- und Behandlungsleitlinien der wissenschaftlich-medizinischen Fachgesellschaften einfließen. Mit der Präsentation aktueller Studiendaten wurde auch ein Ausblick in kurz- und mittelfristig anstehende Weiterentwicklungen gegeben. Das ist es eben, was die Diabetes-Update-Veranstaltungen ihren Teilnehmern bieten: immer einen Schritt voraus $\mathrm{zu}$ sein.

In diesem Heft werden 5 interessante Vorträge als Publikation angeboten, alle verbunden mit der persönlichen Sichtweise und Interpretation der Studiendaten und konkreten Empfehlungen durch erfahrene Experten.

Herr Professor Roland Schmieder, Nephrologe und Hypertensiologe vom Universitätsklinikum Erlangen-Nürnberg, präsentiert zusammen mit seiner Mitarbeiterin Iris Kistner neue Erkenntnisse in der Hochdruckbehandlung. Was mich an diesem Artikel beeindruckt, sind die zahlreichen Hintergrundinformationen und die Detailkritik von Studien mit wichtigen klinischen Implikationen, z. B. zu den Vorteilen der Verwendung thiazidähnlicher Diuretika.

Herr Professor Tom Lindner, Nephrologe aus dem Universitätsklinikum Leipzig, stellt neue Studienergebnisse zum Thema Diabetes und Niere vor. In seinem Artikel weist er insbesondere darauf hin, dass die Diagnose einer diabetischen Nephropathie meist nicht durch histologische Befunde untermauert ist und in einem beträchtlichen Teil der Fälle dann doch eine nichtdiabetische Nephropathie vorliegt.

Herr Priv.-Doz. Dr. Michael Lehrke, Kardiologe aus dem Universitätsklinikum Aachen, stellt die Highlights zum Thema Diabetes und Herz vor. Dabei präsentiert er die neuesten Studiendaten zu diesem Thema sehr souverän aus der Sicht des Kardiologen und gibt konkrete Hinweise für das praktische Vorgehen. Neben der koronaren Herzerkrankung (KHK) ist nun die Herzinsuffizienz zunehmend in den Fokus der diabetologisch interessierten Kardiologen gerückt.

Herr Professor Manfred Dreyer, niedergelassener Diabetologe aus Hamburg, präsentiert die wichtigsten Neuerungen zum Typ-1-Diabetes. Besonders bemerkenswert sind die von ihm beschriebenen Fortschritte auf dem Gebiet der subkutanen Insulininfusion durch Kopplung der Insulinpumpen mit dem kontinuierlichen Glukosemonitoring und die sensorunterstützte Pumpentherapie mit integrierter Alarmfunktion. Diese Systeme werden immer robuster, sodass nun schon zahlreiche Patienten mit Typ-1Diabetes davon profitieren.

Herr Professor Dieter Braus, Chef der Psychiatrie der HELIOS Dr. HorstSchmidt-Kliniken in Wiesbaden, gibt eine sehr lesenswerte aktuelle Übersicht zum Thema Diabetes und Depression. Er zeigt, dass sowohl die Prävention von Typ-2-Diabetes bei depressiven Patienten als auch eine konsequente Behandlung der depressiven Störung bei Typ-2Diabetes relevant sind. Bemerkenswert scheint mir seine Aussage, dass auch ein Diabetologe nach der Aufdeckung einer Depression nicht abwarten, sondern gleich mit einem Antidepressivum behandeln sollte.

\section{Highlights zum Typ-2-Diabetes}

Herr Prof. Michael Stumvoll vom Universitätsklinikum Leipzig stellte neue Daten zur Pathogenese des Typ-2-Diabetes und insbesondere auch zur antihyperglykämischen Behandlung vor. Zunächst das Wichtigste vorweg:

\section{Die Ergebnisse von} EMPA-REG OUTCOME eine Meilensteinstudie in der Diabetologie

In dieser prospektiven randomisierten plazebokontrollierten Outcome-Studie bei 7020 Menschen mit Typ-2-Diabetes sollte eine "Nichtunterlegenheit" (primäres Ziel) oder gar Überlegenheit des SGLT2-Hemmers Empagliflozin im Sinne einer Ereignisreduktion (sekundäres Ziel) gegenüber Plazebo untersucht werden. Empagliflozin, $10 \mathrm{mg}$ oder $25 \mathrm{mg} / \mathrm{Tag}$, wurde im Vergleich zu Plazebo untersucht (1:1:1-Design). Die Studiendauer betrug 3,1 Jahre. Alle Teilnehmer hatten kardiovaskuläre Erkrankungen in der Vorgeschichte, davon nahezu $50 \%$ einen Herzinfarkt. Der primäre Endpunkt war definiert als eine Kombination aus kardiovaskulärem Tod und nichttödlichem Myokardinfarkt oder Schlaganfall. Sekundäre Endpunkte waren u. a. Krankenhauseinweisungen wegen Herzinsuffizienz und die Gesamtmortalität. Eine Behandlung mit Empagliflozin reduzierte das Auftreten des primären Endpunkts um $14 \%$, was 
insbesondere auf eine Reduktion kardiovaskulärer Todesfälle um $38 \%$ zurückzuführen war. Weder das Auftreten von Myokardinfarkten noch Schlaganfällen wurde unter Empagliflozin signifikant verändert. Es kam aber zu einer $\mathrm{Ab}$ nahme der Gesamtmortalität um $32 \%$. Als Nebenwirkungen traten unter Empagliflozin mehr Genitalinfektionen auf (6,8 \% vs. $1,8 \%$ ), die jedoch ausnahmslos unkompliziert verliefen [34].

Die Gründe für diese eindrucksvollen Resultate sind Gegenstand intensiver Diskussionen und Subanalysen. Der Effekt war schon nach 2-4 Monaten sichtbar. Durch die $\mathrm{HbA}_{1 \mathrm{c}}$-Senkung (0,54\% unter $10 \mathrm{mg}$ bzw. 0,6\% unter $25 \mathrm{mg}$ Empagliflozin binnen 12 Wochen) kann dies nicht erklärt werden. Die Ergebnisse von EMPA-REG legen vielmehr eine blutzuckerunabhängige Wirkung des Präparats nahe. Es ist bemerkenswert, dass Empagliflozin eine Senkung des systolischen Blutdrucks um $4 \mathrm{mmHg}$ bewirkte. Nach diesen Daten wird dem Einsatz von Empagliflozin ein fester Platz bei der medikamentösen Therapie des Typ-2-Diabetes einzuräumen sein. Inzwischen wurde auch belegt, dass die Behandlung mit Empagliflozin beim Typ-2-Diabetes im Vergleich zu Plazebo mit einer langsameren Progression einer Nephropathie und einer geringeren Rate an klinisch relevanten Nierenkomplikationen verbunden ist [32]. Ob die oben beschriebenen positiven Effekte auch durch andere SGLT2Hemmer erreicht werden, kann aufgrund der derzeitigen Datenlage nicht beurteilt werden.

\section{Der Wermutstropfen: euglykämische Ketoazidose unter SGLT2-Hemmern}

Im vergangenen Jahr wurden mehrere Arbeiten veröffentlicht, in denen das Auftreten einer euglykämischen diabetischen Ketoazidose (DKA) unter Behandlung mit SGLT2-Inhibitoren beschrieben wurde. In der Publikation von Erondu et al. [8] wurden insgesamt 17.596 Probanden aus randomisierten Studien untersucht. Bei 12 Patienten war eine euglykämische DKA berichtet worden, darunter bei $4(0,04 \%)$ unter $100 \mathrm{mg}$, bei $6(0,11 \%)$ unter $300 \mathrm{mg}$
Canagliflozin und bei $2(0,03 \%)$ unter einer Vergleichssubstanz, entsprechend einer Inzidenzrate von 0,5 bzw. 0,76 bzw. 0,24 pro 1000 Patientenjahre. Die meisten dieser Patienten hatten beim Auftreten der DKA einen Blutglukosespiegel $>300 \mathrm{mg} / \mathrm{dl}$, standen unter einer Insulintherapie und hatten für eine DKA prädisponierende Grunderkrankungen wie einen Typ-1-Diabetes. Es handelte sich also um Personen, die einen Insulinmangel aufwiesen und den SGLT2Hemmer außerhalb der erlaubten Indikation erhielten. Solche Komplikationen können wie in anderen Fällen der DKA z. B. durch Stress oder hohen Alkoholkonsum ausgelöst werden und zeichnen sich durch allgemeines Krankheitsgefühl und Übelkeit aus. Die Empfehlung des Referenten ist, in solchen Fällen die Ketonkörper zu messen und dann Flüssigkeit, Kohlenhydrate und Insulin zuzuführen.

\section{Triple-Therapie mit Metformin, DPP-4-Hemmer und SGLT2-Inhibitor}

Die Behandlung des Typ-2-Diabetes mit Metformin, einem DPP-4-Hemmer und SGLT2-Inhibitoren per se ist nicht mit einem gehäuften Auftreten von Hypoglykämien verbunden. Die Behandlung mit den 3 Medikamentenklassen war Gegenstand von 2 großen Studien aus dem letzten Jahr $[20,27]$. In der Studie von Matthaei et al. [20] wurden bei 315 Probanden mit Typ-2-Diabetes die Wirksamkeit und Sicherheit einer TripleTherapie mit Saxagliptin (SAXA) oder Plazebo zusätzlich („add-on“) zu einer vorbestehenden Therapie mit Metformin (MET) plus Dapagliflozin (DAPA) untersucht. Nach 24 Wochen zeigte sich eine signifikant größere $\mathrm{HbA}_{1 \mathrm{c}}$-Reduktion unter SAXA $(-0,51 \%)$ vs. Plazebo $(-0,16 \%)$. Unter der Triple-Therapie erreichten $35 \%$, unter Zweifachtherapie $23 \%$ einen $\mathrm{HbA}_{1 \mathrm{c}}$ von unter $7 \%$. Die Nebenwirkungen waren vergleichbar. In einer Studie von Rosenstock et al. [27] wurde bei 534 metforminbehandelten Probanden mit einem unter alleiniger Metformintherapie schlecht eingestellten Typ-2-Diabetes (mittlerer $\mathrm{HbA}_{1 \mathrm{c}}$ : 8,9\%, Diabetesdauer: 7,6 Jahre, BMI: 32 kg/m²) zusätzlich SAXA+DAPA gegeben und mit einem Einzel-Add-On SAXA oder DAPA verglichen. Nach 24 Wochen war der $\mathrm{HbA}_{1 \mathrm{c}}$ unter der Triple-Therapie um $-1,5 \%$ reduziert, unter MET+DAPA um $-1,2 \%$, unter MET+SAXA um -0,9\%. Unter der Triple-Therapie erreichten $41 \%$, unter MET+DAPA $22 \%$ und unter MET+SAXA $18 \%$ einen $\mathrm{HbA}_{1 \mathrm{c}}$ von unter $7 \%$. In beiden oben beschriebenen Studien waren Hypoglykämien selten, schwere Hypoglykämien traten nicht auf. Die Triple-Therapie funktioniert also, sie ist wirksamer als die Zweifachbehandlung, ohne dass mehr Nebenwirkungen $\mathrm{zu}$ verzeichnen sind.

\section{Highlights zu Pathophysiologie und Prävention}

Die aktuellen wissenschaftlichen Arbeiten zu diesem Thema wurden von Herrn Prof. Stefan Martin vom Westdeutschen Diabetes- und Gesundheitszentrum Düsseldorf vorgestellt.

\section{Effekt einer sehr niedrigkalorischen Kost vergleichbar mit bariatrischer Chirurgie}

Bereits in einer Arbeit aus dem Jahre 2011 wurde exemplarisch gezeigt, dass eine sehr kalorienarme Kost (600 kcal/Tag) bei Menschen mit einem weniger als 4 Jahre bekannten Typ-2-Diabetes schon nach wenigen Wochen zu einer Normalisierung des Kohlenhydratstoffwechsels und binnen 8 Wochen zu einer Remission des Diabetes mit Normalisierung der Insulinsekretion führt. Die gleiche Arbeitsgruppe beschrieb nun die Effekte einer 8-wöchigen Formuladiät mit $600 \mathrm{kcal} / \mathrm{Tag}$ bei Patienten mit einer Diabetesdauer von weniger als 4 Jahren im Vergleich zu Patienten mit einer Diabetesdauer von mehr als 8 Jahren. In beiden Gruppen kam es binnen 8 Wochen $\mathrm{zu}$ einer Gewichtsabnahme von etwas mehr als $14 \%$ des Ausgangsgewichts. Die Blutzuckersenkung war bei den Patienten mit kurzer Diabetesdauer einheitlich dramatisch wie in der Studie von 2011. In der Gruppe mit längerer Diabetesdauer war allerdings der Effekt der drastischen Kalorienreduktion auf den Blutzuckerspiegel und die Insu- 
linsekretion heterogen; einige Teilnehmer reagierten vergleichbar wie die der Gruppe mit kurzer Diabetesdauer, einige langsamer, andere überhaupt nicht. Insgesamt erreichten nach 8 Wochen $87 \%$ in der Gruppe mit kurzer Diabetesdauer und $50 \%$ in der Gruppe mit längerer Diabetesdauer nichtdiabetischen Werte. Unabhängig von der Diabetesdauer wurden in beiden Gruppen klinisch signifikante Verbesserungen von Blutdruck und Blutlipidwerten erzielt [31]. Diese Effekte sind durchaus mit denen der bariatrischen Chirurgie vergleichbar.

\section{Rasche Entwicklung einer Insulinresistenz nach mehreren Schlemmermahlzeiten}

Aus einer kürzlich publizierten experimentellen Untersuchung bei gesunden Freiwilligen haben wir gelernt, dass die Zufuhr von $6000 \mathrm{kcal} / \mathrm{Tag}$ entsprechend einer exzessiven Kalorienzufuhr schon nach wenigen Tagen zu einer Oxidation und Carbonylierung des Glukosetransporters 4 (GLUT-4) und zur Insulinresistenz führt. All-you-can-eat-Angebote sind also dem Stoffwechsel nicht zuträglich [4]. Der negative Effekt tritt rasch zu Tage. Der Referent wies darauf hin, dass dieser Versuch durchaus mit der Nahrungsaufnahme bei einer durchschnittlichen Kreuzfahrt vergleichbar ist.

\section{Schützt Schlaf vor Diabetes?}

Zur Frage der optimalen Schlafdauer und deren Beziehung zum Diabetesrisiko gibt es unterschiedliche Angaben in der Literatur. Dazu wurde nun eine Metaanalyse unter Einschluss von 10 prospektiven Studien publiziert. Insgesamt wurden 482.500 Teilnehmer mit einem Followup von 2,5 bis zu 16 Jahren analysiert. Es wurden 18.443 neue Fälle von Diabetes registriert. Dabei zeigte sich, dass es einen U-förmigen Zusammenhang gibt zwischen Schlafdauer und dem Risiko, einen Typ-2-Diabetes zu entwickeln. Das geringste Risiko liegt bei einer Schlafdauer zwischen 7 und $8 \mathrm{~h}$. Sowohl eine kürzere als auch eine längere Schlafdauer sind mit einem signifikanten Anstieg des Risikos für Typ-2-Diabetes verbunden [29]. Der Referent wies darauf hin, dass die optimale Schlafdauer individuell sehr unterschiedlich ist, und es auch Personen gibt, die nach 5-6 h gut ausgeschlafen sind.

\section{Highlights zum Thema Diabetes und Schwangerschaft}

Dr. Helmut Kleinwechter, Betreiber einer großen Diabetes-Schwerpunktpraxis in Kiel, referierte zum Thema Diabetes und Schwangerschaft. Als Vorsitzender des Ausschusses Diabetes und Schwangerschaft und der entsprechenden Leitlinienkommission der DDG hat seine Sichtweise ein ganz besonderes Gewicht. Aus der Fülle der von ihm vorgestellten Studien und wertvollen Kommentare sollen hier 3 herausgehoben werden:

\section{Metformin ist zur Behandlung adipöser Schwangeren obsolet}

Es war angenommen worden, dass eine Behandlung adipöser Schwangeren mit Metformin deren Körpergewicht und damit das Risiko der Kinder für Adipositas und frühzeitige Mortalität reduziert. $\mathrm{Zu}$ diesem Thema wurde nun eine randomisierte, doppelblinde plazebokontrollierte multizentrische Studie aus 15 geburtshilflichen Zentren aus Großbritannien publiziert bei der 449 Schwangere mit einem Body-Mass-Index von mindestens $30 \mathrm{~kg} / \mathrm{m}^{2}$ (mittlerer BMI: $38 \mathrm{~kg} / \mathrm{m}^{2}$ ) eingeschlossen waren. Darunter waren auch Frauen mit PCO-Syndrom. Eine Gruppe erhielt ab der 12.-16. Schwangerschaftswoche (SSW) bis zur Geburt bis zu $2500 \mathrm{mg}$ Metformin/Tag, die andere Plazebo. Zwischen den beiden Gruppen ergab sich kein signifikanter Unterschied der mittleren Geburtsgewichte, der Abortrate, von Schwangerschaftsabbrüchen, Totgeburten, Sectioraten, Frühgeburten oder dem Auftreten eines Gestationsdiabetes. Auch ergaben sich in der 36. SSW keine Unterschiede beim Insulinresistenzparameter HOMA-IR und bei den Nüchtern- und 2-h-Blutglukosewerten im oralen Glukosetoleranztest (oGTT). In der Metformingruppe traten signifikant häufiger Übelkeit und Durchfall auf [7].

Der Referent stellte als Fazit für die Praxis heraus, dass sich die Verordnung von Metformin bei adipösen Schwangeren mit normaler Glukosetoleranz nicht zur Verbesserung von Schwangerschaftsverlauf und -ausgang eignet und unterbleiben sollte. Dies gilt auch für Frauen mit PCO-Syndrom.

\section{Hypertonie ist bei Schwangeren ein Fehlbildungsrisiko}

Die Frage, ob Hypertonie bei Schwangeren ein Fehlbildungsrisiko darstellt, wurde anhand der Registerdaten der Krankenakten des amerikanischen Gesundheitsvorsorgeprogramms Medicaid bei 878.126 Schwangerschaften untersucht. In 19.789 Fällen (2,35\%) bestand eine chronische arterielle Hypertonie; davon wurden 8307 (42\%) antihypertensiv behandelt. Fehlbildungen traten bei 3,49\% der Schwangeren ohne Hochdruck, bei 5,91\% mit behandeltem und 5,06\% mit unbehandeltem Hochdruck auf. Sowohl unbehandelter als auch behandelter Hochdruck war mit einem um 20-30 \% erhöhten Risiko für eine kindliche Fehlbildung, insbesondere Herzfehlbildungen, verbunden [3]. In der Studie wurde allerdings nicht analysiert, ob die Blutdruckziele bei der Behandlung erreicht wurden und welchen Einfluss die eingesetzten Medikamente hatten. Der Referent betonte, dass die Hypertonie bisher nicht allgemein als Fehlbildungsrisiko angesehen worden war. Darauf sollte nun im Rahmen der Perinataldiagnostik besonders geachtet werden.

\section{Pharmakotherapie des \\ Gestationsdiabetes (GDM) mit Glibenclamid ist risikoreich}

Nach bisheriger Studienlage galt Glibenclamid als sicher zur Behandlung des GDM. Diese Aussage stützte sich aber auf sehr limitierte Daten aus 3 Interventionsstudien mit insgesamt 268 Schwangeren weltweit. Während die Glibenclamidbehandlung des GDM in den USA weit verbreitet ist und inzwischen die Insulintherapie des GDM überholt hat, hat die Deutsche Diabetes-Gesellschaft (DDG) Glibenclamid bei GDM aufgrund der dünnen Datenlage abgelehnt. Diese Haltung der DDG wird nun durch die 
Auswertung des bevölkerungsbezogenen Registers von Betriebskrankenkassen der USA aus dem Zeitraum von 2000-2011 unterstützt. Von 110.879 Schwangeren mit GDM erhielten 9173 (8,3\%) entweder Glibenclamid ( $n=4982$ ) oder Insulin $(n=4191)$. Nach entsprechenden Adjustierungen ergaben sich für Schwangere mit Glibenclamidtherapie die folgenden signifikant erhöhten relativen Risiken (RR) gegenüber der Insulintherapie: Aufnahme auf neonatale Intensivstation: RR 1,41. Atemnotsyndrom: RR 1,63. Hypoglykämie: RR 1,40. Geburtstrauma: RR 1,35. LGA (Kind zu groß bezogen auf das Gestationsalter): RR 1,43 [6]. Diese Daten werden in den USA und international dazu führen, die Glibenclamidtherapie des GDM zu hinterfragen und ggf. auf bestimmte Subgruppen zu limitieren. Der Referent kam für die Praxis zu dem Schluss, dass die Therapie des GDM mit Glibenclamid mit nicht unerheblichen Risiken behaftet ist und dass diese auch weiterhin von der DDG nicht empfohlen werden sollte.

\section{Highlights zum diabetischen Fuß}

Neue Studien zum diabetischen Fuß wurden von Herrn Prof. Maximilian Spraul vom Matthias-Spital Rheine vorgestellt und diskutiert; davon hier meine Auswahl:

\section{Das „Aus" für die HBO-Therapie beim diabetischen Fußsyndrom}

Seit mehr als 20 Jahren wird die hyperbare Sauerstofftherapie (HBO) bei der Behandlung diabetischer Fußulzera eingesetzt. Ältere Studien zu diesem Thema sind mit erheblichen Mängeln des Studiendesigns behaftet. Eine neue Studie von 2016 [9], eine Cochrane-Übersichtsarbeit von 2015 [18] und die aktualisierte Leitlinie des „National Institute of Health and Care Excellence" (NICE) in Großbritannien [21] kamen alle zu dem gleichen Ergebnis: Eine HBO-Therapie ist für die Reduzierung der Amputationsrate und die Verbesserung der Wundheilung bei diabetischen Fußulzera untauglich. Die Studie von Fedorko et al. [9] kann als wegweisend angesehen werden: In der dop- pelblinden randomisierten Studie wurden Patienten mit diabetischen Fußulzera der Wagner-Stadien 2-4 untersucht. Zusätzlich zurStandardtherapie erhielten sie entweder 30 Sitzungen einer $\mathrm{HBO}$ von 90 min Dauer mit einem Überdruck von $244 \mathrm{kPa}$ oder eine Plazebotherapie mit 30 Sitzungen in der gleichen Überdruckkammer mit Atemluft bei einem Druck von $125 \mathrm{kPa}$. Nach 12 Wochen bestand auf der Basis einer verblindeten Beurteilung durch einen Gefäßchirurgen bei 11 von 49 Patienten in der mit $\mathrm{HBO}$ und 13 von 54 in der mit Plazebo behandelten Gruppe eine Indikation zur Amputation. Auch bezüglich der verschiedenen Parameter der Wundheilung fand sich kein Unterschied zwischen den beiden Gruppen. Nach Meinung des Referenten sollte die HBO-Therapie nicht mehr zur Behandlung des diabetischen Fußsyndroms eingesetzt werden, weil diesbezüglich für diese teure Behandlung kein Effektivitätsnachweis vorliegt und sie sogar schädlich sein kann.

\section{Risikofaktoren für eine Amputation bei infizierten Fußulzera}

Die Infektion eines diabetischen Fußulkus ist eine häufige Komplikation. In der sog. Eurodiale-Studie sollte herausgefunden werden, ob sich bei infizierten Ulzera Risikofaktoren identifizieren lassen, die potenziell zu einer Amputation führen. Dabei wurden 575 Patienten mit neuentdeckten Fußulzera aus 14 Fußzentren in Europa monatlich nachuntersucht und die kompletten Datensätze analysiert. Bei 159 (28 \%) Patienten wurde im Nachuntersuchungszeitraum von $1 \mathrm{Jahr}$ eine Amputation durchgeführt; 126 Minor- und 33 Majoramputationen. Als unabhängige Risikofaktoren für eine Amputation wurden folgende Parameter identifiziert: Ödeme um die Wunde, fauliger Geruch, Exsudat aus der Wunde, tiefes Ulkus mit Knochenkontakt, Fieber und ein erhöhtes CRP. Außerdem waren das Vorliegen einer PAVK und der Schweregrad der Infektion mit einer Amputation assoziiert [22].

Die Autoren entwickelten aus den o. g. Daten einen Score für das Amputationsrisiko, dessen Zuverlässigkeit allerdings noch in weiteren Studien zu prüfen ist.

\section{Neues zur diabetischen Neuropathie}

Herr Prof. Dan Ziegler vom Deutschen Diabeteszentrum in Düsseldorf stellte neue Studien zum Thema diabetische Neuropathie vor, von denen hier 3 besonders interessante ausgewählt wurden:

\section{Durch Insulintherapie induzierte Neuropathie}

Die Insulinneuritis ist eine relativ seltene Erkrankung, die jedoch jeder Diabetologe kennen sollte, weil sie meist in der Therapiephase direkt nach der Diagnosestellung des Diabetes auftritt. Es handelt sich dabei um eine durch die Insulintherapie induzierte schmerzhafte Neuropathie im Sinne einer Small-Fiber-Neuropathie, die durch eine ausgeprägte und schnelle Verbesserung der Diabeteseinstellung verursacht wird.

In einer retrospektiven Untersuchung bei 954 Patienten mit akuten neuropathischen Schmerzen erfüllten $11 \%$ die Kriterien einer therapieinduzierten Neuropathie bei Diabetes (TIND). Dabei bestand $u$.a. eine starke Korrelation zwischen der Abnahme des $\mathrm{HbA}_{1 \mathrm{c}}$-Werts und dem Ausmaß der neuropathischen Schmerzen sowie dem Ausmaß der sympathoadrenergen Dysfunktion mit Blutdruckabfall am Kipptisch. Bei einem Abfall des $\mathrm{HbA}_{1 \mathrm{c}}$ um 2-3\% in 3 Monaten lag das absolute Risiko für eine TIND bei $20 \%$, bei Abfall um $>4 \%$ betrug es über $80 \%$ [13].

In Anbetracht der Seltenheit der TIND ließ der Referent die Frage offen, ob die vorliegenden Daten eine rasche und starke Absenkung des $\mathrm{HbA}_{1 \mathrm{c}}$ mit Insulin verbieten.

\section{Behandlung neuropathischer} Schmerzen mit Capsaicinpflaster (8\%)

Eine neue Möglichkeit für die Behandlung neuropathischer Schmerzen ist die Anwendung von Capsaicin in Form eines Capsaicinpflasters (8\%ige Konzentration; Qutenza $\left.{ }^{\circledR}\right)$, das nun auch für die diabetische Neuropathie zugelassen ist. Dadurch werden die kutanen Nozizeptoren weniger empfindlich für verschiede- 
Hier steht eine Anzeige.

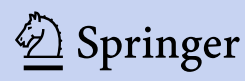


Hier steht eine Anzeige.

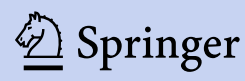


ne Schmerzreize. Das schmerzhafte Areal wird vom Arzt auf der intakten, nichtgereizten Haut markiert und das Pflaster auf die entsprechende Größe zugeschnitten. Die Applikationsdauer beträgt $30 \mathrm{~min}$. In einer offenen randomisierten multizentrischen Studie bei 559 Patienten mit neuropathischen Schmerzen war Qutenza ${ }^{\circledR}$ $(n=282)$ der systemischen Therapie mit Pregabalin $(n=277)$ bezüglich Schmerzrückgang und Zeit bis zu dessen Beginn nicht unterlegen. Systemische Nebenwirkungen traten unter Qutenza ${ }^{\circledR}$ im Gegensatz zu Pregabalin praktisch nicht auf [14]. Die entsprechenden bei Diabetespatienten durchgeführten Studien wurden allerdings noch nicht publiziert.

\section{Testosterongel zur Verbesserung der Sexualfunktion unwirksam}

Testosteron wird bei älteren Männern zunehmend appliziert. In einer plazebokontrollierten Studie wurde die Frage geprüft, ob die Gabe von Testosteron bei älteren Männern die Progression einer Arteriosklerose hemmt und positive Wirkungen auf die Sexualfunktion hat. 308 Männer im Alter von $\geq 60$ Jahren (mittleres Alter: 67,6 Jahre) mit niedrigen oder niedrig normalen Testosteronspiegeln (100-400 ng/dl, freies Testosteron: $<50 \mathrm{pg} / \mathrm{ml}$ ) erhielten entweder täglich 7,5 g eines 1\%igen Testosterongels oder Plazebo. Die Dosis des Gels wurde auf einen Zielbereich des Testosteronspiegels von 500-900 ng/dl titriert. Nach einer Studiendauer von 3 Jahren zeigte sich zwischen den beiden Gruppen kein signifikanter Unterschied bezüglich der Änderung der Intima-Media-Dicke, des Kalzium-Scores der Koronararterien, der Libido, der Erektionsfähigkeit, der allgemeinen Sexualfunktion und der Lebensqualität. Hämatokrit und PSA-Werte stiegen unter Testosteron stärker an [2]. Nach den Ergebnissen dieser sauber durchgeführten klinischen Studie ist nach Ansicht des Referenten die Verordnung von Testosterongel bei niedrig-normalem Testosteronspiegel nicht mehr gerechtfertigt. Davon unberührt bleibt natürlich die Notwendigkeit zur Testosteronsubstitution bei Hypogonadismus.

\section{Highlights zu Ernährung und Diabetes}

Herr Prof. Andreas Pfeiffer, Direktor am Deutschen Institut für Ernährungsmedizin (DIFE) und der Charité Universitätsmedizin Berlin, stellte neue Studien zum Thema Ernährung vor. Daraus hier die m. E. 3 wichtigsten Arbeiten:

\section{Eierangereicherte Kost für Lipidstoffwechsel bei Typ-2- Diabetes unbedenklich}

Bisherige Studien kamen bezüglich der Empfehlungen für den Konsum von Eiern bei Typ-2-Diabetes zu widersprüchlichen Ergebnissen. In einer randomisierten, kontrollierten Studie wurde nun untersucht, ob eine eireiche Diät (2 Eier pro Tag für 6 Wochen) im Kontext einer Diät mit einfach und mehrfach ungesättigten Fettsäuren verglichen mit einer eiarmen Diät (weniger als 2 Eier pro Woche) Triglyzeride, HDL-Cholesterin, Gesamtcholesterin sowie das Gewicht bei Menschen mit Übergewicht, Prädiabetes oder Typ-2-Diabetes beeinflusst. Dazu wurden 140 Studienteilnehmer randomisiert und über 3 Monate mit einer der 2 Diäten behandelt. Beide Gruppen erhielten die gleiche Proteinmenge. Nach 3 Monaten zeigten sich bei allen o. g. Lipidparametern und auch bezüglich der Blutglukosespiegel keine Unterschiede. Die Gruppe mit der eireichen Diät zeigte geringeren Hunger und eine bessere Sättigung nach dem Frühstück [11]. Auch andere Studien ergaben, dass eine erhöhte Aufnahme von Cholesterin bei den meisten Menschen nicht zu einem Anstieg des Cholesterinspiegels führt. Der Referent erläuterte dies anhand der Physiologie des Lipidstoffwechsels und schlussfolgerte, dass es nach dem aktuellen Kenntnisstand keinen Grund gibt, einen zurückhaltenden Konsum von Eiern zu empfehlen.

\section{Verzicht auf das Frühstück ungünstig bei Typ-2-Diabetes}

Viele Menschen mit Typ-2-Diabetes lassen das Frühstück aus und meinen, dass sie damit den Diabetes günstig beeinflussen. Das Gegenteil ist aber der Fall. In einer Studie bei 22 Probanden mit
Typ-2-Diabetes (mittleres Alter: 56 Jahre, Diabetesdauer: 8,4 Jahre, $\mathrm{HbA}_{1 \mathrm{c}}$ : 7,7\%) wurde in einem sog. Cross-Over-Design an 2 Tagen einmal das Frühstück eingenommen und das zweite Mal weggelassen und jeweils der Einfluss auf die postprandialen Glukosewerte, Insulin, C-Peptid, freie Fettsäuren, Glukagon und GLP-1Werte nach dem Mittag- und nach dem Abendessen untersucht. Es zeigte sich, dass das Auslassen des Frühstücks zu erhöhten Werten von Glukose, freien Fettsäuren und Glukagon und einer verminderten Sekretion von Insulin und GLP-1 nach dem Mittag- und Abendessen führte. An dem Tag ohne Frühstück war zudem der Maximalwert von Insulin sowohl nach dem Mittagessen als auch nach dem Abendessen um 30 min verzögert [16]. Das Frühstück scheint also zu einer Sensibilisierung der $\beta$-Zellen für die glukoseinduzierte Insulinsekretion zu führen. Der Referent sieht dies als ein weiteres Argument dafür, Patienten mit Typ2-Diabetes die regelmäßige Einnahme eines Frühstücks zu empfehlen.

\section{Was ist die günstigste}

Speisenabfolge bei Typ-2-Diabetes

Die Reihenfolge der Nahrungsbestandteile Gemüse, Protein, Kohlenhydrate hat einen deutlichen Einfluss auf die postprandialen Spiegel von Glukose und Insulin. Das ergab eine Studie bei metforminbehandelten Probanden mit Typ-2Diabetes. 11 Teilnehmer (mittleres Alter: 54 Jahren BMI: $33 \mathrm{~kg} / \mathrm{m}^{2}$ ) erhielten eine isokalorische Mahlzeit von $628 \mathrm{kcal}$ mit 55 g Protein, 68 g Kohlenhydraten, $16 \mathrm{~g}$ Fett, mit derselben Komposition an 2 verschiedenen Tagen. Die Reihenfolge am ersten Tag war: zuerst KH (Ciabattabrot und Orangensaft), 15 min später Protein (gegrillte Hühnerbrust) und Gemüse (Blattsalat, Tomatensalat mit fettarmer Vinaigrette, gekochtem Broccoli und Butter). Diese Reihenfolge wurde bei dem Test 1 Woche später umgekehrt. Wenn zuerst Gemüse und Protein gegessen wurden, lagen die Glukosewerte nach 30 und $60 \mathrm{~min}$ um $29 \%$ bzw. $37 \%$ tiefer, und die postprandialen Insulinspiegel waren im Vergleich zu der mit den Kohlenhydraten beginnenden Reihenfolge niedriger [30]. Aus wissen- 
schaftlichen Gründen wäre daher Patienten mit Typ-2-Diabetes zu empfehlen, zum Mittag- und Abendessen zunächst Gemüse und Protein und dann erst die Kohlenhydrate zu konsumieren.

\section{Hot Topic Lipidologie}

Herr Prof. Klaus Parhofer vom Klinikum Großhadern der LMU München stellte die neuesten Ergebnisse aus der Lipidologie vor, deren klinisch bedeutsamsten Resultate hier referiert werden sollen:

\section{Kombination von Ezetimib und Statinen ist sehr wirksam und sicher}

Wenn mit einer Statintherapie die Zielwerte von LDL-Cholesterin nicht zu erreichen waren oder Statine nur in einer geringen Dosis vertragen wurden, wurde schon seit 10 Jahren Ezetimib eingesetzt. Es war aber bisher noch unklar, ob sich die durch Ezetimib bewirkte Senkung des LDL-Cholesterins auch in einer Reduktion kardiovaskulärer Ereignisse niederschlägt. Letzteres wurde nun in der IMPROVE-IT-Studie belegt [5].

In dieser doppelblinden, randomisierten kontrollierten Studie wurden mehr als 18.000 Patienten mit Zustand nach akutem Koronarsyndrom untersucht. Die eine Gruppe erhielt Simvastatin, die andere Simvastatin+Ezetimib. Die erste Gruppe hatte am Studienende einen LDL-Cholesterin-Wert von 69,5 mg/dl, die Gruppe mit Simvastatin+Ezetimib $53,4 \mathrm{mg} / \mathrm{dl}$. Nach einer Studienlaufzeit von 7 Jahren fand sich ein signifikanter Vorteil der kombiniert behandelten Gruppe bezüglich des primären Endpunkts (kardiovaskulärer Tod, nichttödlicher Herzinfarkt, nichttödlicher Schlaganfall, instabile Angina pectoris, kardiovaskuläre Revaskularisation). Hinsichtlich der Nebenwirkungsrate gab es keinen Unterschied. Der Referent betonte, dass diese Studie von sehr großer Bedeutung ist, weil sie mehrere wichtige Fragen in der Lipidologie beantwortet:

- Eine Absenkung des LDL-Cholesterin-Spiegels von knapp $70 \mathrm{mg} / \mathrm{dl}$ auf $54 \mathrm{mg} / \mathrm{dl}$ überträgt sich in eine weitere Reduktion der kardiovaskulären Ereignisrate.
- Die LDL-Senkung kann auch über einen Nichtstatinansatz erfolgen.

- Ezetimib ist sicher.

\section{Hohe Wirksamkeit und Sicherheit von PCSK9-Inhibitoren zur Senkung des LDL-Cholesterin- Spiegels}

Der Einsatz monoklonaler Antikörper gegen PCSK9 führt zu einer dramatischen Absenkung des LDL-Cholesterins und gilt als einer der größten Fortschritte in der Lipidologie. Dazu wurden 2015 zwei große Studien publiziert. Im Rahmen einer randomisierten doppelblinden plazebokontollierten Phase-3-Studie wurden die Wirksamkeit und Sicherheit von Aliroculab überprüft. Dazu wurde bei 2341 Patienten mit einem hohen oder sehr hohen kardiovaskulären Risiko entweder $150 \mathrm{mg}$ Aliroculab oder Plazebo über 72 Wochen hinweg alle 2 Wochen subkutan injiziert. Aliroculab resultierte in einer hochsignifikanten 62\%igen $\mathrm{Ab}$ senkung des LDL-Cholesterin-Werts, die bereits über den kurzen Zeitraum von 1,5 Jahren zu einer verminderten Rate an kardiovaskulären Ereignissen führte [25].

Im Rahmen einer unverblindeten randomisierten Studie wurden 4465 Patienten, die an Phase-2- oder Phase-3-Studien mit Evolocula teilgenommen hatten, nach Abschluss der Originalstudien in eine Evoloculab- oder eine Kontrollgruppe randomisiert und weiter beobachtet. Die Teilnehmer der Evoloculabgruppe erhielten entweder 140 mg Evoloculab s. c. alle 2 Wochen oder $420 \mathrm{mg}$ s. c. monatlich. Im Vergleich zur Standardtherapie fand sich unter Evoloculab eine zusätzliche LDL-Absenkung von $61 \%$ (Mittelwert von $120 \mathrm{mg} / \mathrm{dl}$ abgesenkt auf $68 \mathrm{mg} / \mathrm{dl}$ ). Über den Studienverlauf von 1 Jahr hatten die mit Evoloculab behandelten Patienten eine signifikant geringere kardiovaskuläre Ereignisrate von $0,95 \%$ im Vergleich zur Kontrollgruppe mit 2,18\% [28].

Der Referent betonte, dass diese Ergebnisse die Basis dafür sind, diese Antikörper bereits jetzt, also noch vor der Verfügbarkeit klassischer Outcome-Studien, bei selektionierten Patienten mit einem sehr hohen kardiovaskulären Ri- siko und schlechten LDL-CholesterinWerten einzusetzen.

\section{Senkung des Spiegels von Lp(a) durch PCSK9-Hemmer}

Erhöhte Spiegel von Lipoprotein(a) stellen bekanntermaßen einen unabhängigen Risikofaktor für kardiovaskuläre Erkrankungen dar. Bisher gab es aber kaum wirksame Therapieansätze, um den Spiegel von $\mathrm{Lp}(\mathrm{a}) \mathrm{zu}$ senken, auch nicht mit Statinen. Nun wurde herausgefunden, dass PCSK9-Antikörper, die primär den Spiegel von LDLCholesterin erniedrigen, auch $\mathrm{zu}$ einer etwa 25\%igen Absenkung der Lp(a)Konzentrationen führen [23]. Es konnte gezeigt werden, dass dieser Abfall von Lp(a) durch PCSK9-Hemmer eine extreme Stimulation der LDL-RezeptorAktivität widerspiegelt [26]. Nach Ansicht des Referenten könnte dies nun für die Therapie nutzbar gemacht werden.

\section{Hot Topic Geriatrie}

Das Thema Geriatrie wurde von Herrn Dr. Wernecke, Internist und Geriater aus Hamburg, als Hot Topic vorgetragen und diskutiert. Es war sehr wichtig, dieses Thema gesondert $\mathrm{zu}$ besprechen, weil ja die meisten Diabetologen auch mit geriatrischen Patienten zu tun haben und sich dabei die Behandlungsprinzipien z.T. wesentlich von den üblichen Vorgehensweisen in der Diabetologie unterscheiden.

\section{Die wichtigsten Beurteilungs- kriterien: Funktionsstörungen (,handicaps") und Gebrechlichkeit ("frailty")}

Der Autor wies insbesondere auf die Probleme der Multimorbidität, Polypharmazie und Arzneimittelinteraktionen und deren Einfluss auf die Behandlungsstrategie hin. Funktionsstörungen („handicaps") spielen für die Beurteilung der Leistungsfähigkeit und der allgemeinen Behandlung dieser Menschen eine große Rolle, weil sie vielfältige Wechselwirkungen mit der diabetischen Stoffwechsellage aufweisen. Eine aktuelle Arbeit mit Versorgungsdaten aus den USA ergab, 
dass der Blutzucker bei multimorbiden Patienten mit komplexen Funktionsstörungen in ca. $60 \%$ der Fälle mit einem $\mathrm{HbA}_{1 \mathrm{c}}$-Wert von unter $7 \%$ viel zu scharf eingestellt ist. Von den Betroffenen standen sogar über $50 \%$ unter einer Insulinund/oder Sulfonylharnstofftherapie, die ja mit einem erhöhten Risiko für schwere Hypoglykämien verbunden ist [19]. Anders als bei jüngeren Patienten ist bei geriatrischen Menschen Behinderung als Resultat von Funktionseinschränkungen für das Mortalitätsrisiko wesentlich entscheidender als die zugrunde liegende Morbidität.

Der Referent wies besonders auf den geriatrischen Begriff "frailty“ (Gebrechlichkeit) hin, der für die Behandlungsintensität sehr relevant ist. Das Vorhandensein von Gebrechlichkeit erhöht nach einer neuen Studie die 1-Jahres-Mortalität von Klinikpatienten von 14 auf $26 \%$ und die Mortalität während des stationären Aufenthalts um den Faktor 3 [12].

Gebrechlichkeit liegt vor wenn mindestens 3 der folgenden 5 Symptome vorhanden sind:

1. ungewollter Gewichtsverlust $>5 \mathrm{~kg}$ in $1 \mathrm{Jahr}$,

2. allgemeine Erschöpfung (von Patienten berichtet),

3. Schwäche, d. h. Abnahme der groben Kraft (Handkraftmessung),

4. langsame Gehgeschwindigkeit (z. B. $5 \mathrm{~m}$ in $>7 \mathrm{~s}$ ),

5. niedriger physischer Aktivitätslevel.

\section{Hot Topic interventionelle Angiologie}

Herr Prof. Dierk Scheinert vom Universitätsklinikum Leipzig stellte den aktuellen Standard und die neuen Möglichkeiten der interventionellen Angiologie vor, die insbesondere auch den Patienten mit einem diabetischen Fußsyndrom zugutekommen.

\section{Prävalenz und Risikofaktoren der PAVK}

In einem systematischen Übersichtsartikel wurde eindrucksvoll beschrieben, wie die Prävalenz der PAVK aufgrund der steigenden Lebenserwartung und durch ungünstige Lebensstilfaktoren weltweit stark zunimmt, wobei sich insgesamt kein Unterschied in der Verteilung zwischen Männern und Frauen zeigt. Rauchen, Diabetes, hoher Blutdruck und eine kardiovaskuläre Erkrankungen in der Anamnese schlagen dabei ganz besonders zu Buche [10]. Im Rahmen der prospektiv angelegten BARI 2D-Studie wurde das Risiko des Auftretens einer PAVK bei 1479 Patienten mit bekanntem Diabetes und anfangs normalem Knöchel-ArmIndex (ABI) untersucht. Trotz einer unter Studienbedingungen optimierten medikamentösen Therapie traten über einen Beobachtungseitraum von 4,6 Jahren bei 20,5\% eine Verschlechterung des ABI sowie die Notwendigkeit für eine Revaskularisationsmaßnahme an Gefäßen der unteren Extremitäten oder einer Amputation auf [1].

\section{Vor Amputation immer Angio- grafie oder Revaskularisations- maßnahme}

Erschreckende Ergebnisse erbrachte eine Analyse der Entlassungsdaten aus deutschen Krankenhäusern. Entsprechend der ICD 10-Kodierung wurden 41.882 Versicherte der Barmer GEK identifiziert, die zwischen 2009 und 2011 wegen PAVK in stationärer Behandlung waren. Zudem wurden Daten bis 2 Jahre vor der Indexhospitalisierung erfasst und eine Nachverfolgung bis zu 4 Jahre durchgeführt. Erwartungsgemäß war die Amputationshäufigkeit bei Patienten mit fortgeschrittener PAVK $(0,5 \%$ im Rutherford-Stadium 1-3, $42 \%$ im Rutherford-Stadium 6) deutlich höher. Auffallend war jedoch, dass bei $44 \%$ aller Fälle vor der Amputation keine Angiografie in der Klinik vorgenommen worden war. Auch bei Berücksichtigung aller in den vorangegangenen 24 Monaten getroffenen stationären und ambulanten Maßnahmen hatten immer noch $37 \%$ aller Amputierten keine Angiografie oder Revaskularisationsmaßnahme erhalten [24]. Ähnlich schlechte Zahlen sind aus den USA bekannt.

Der Referent forderte, dass unbedingt vor jeder Amputation bei chronischen Ulzera eine angiografische Darstellung bzw. (endovaskuläre oder chirurgische)
Revaskularisationsmaßnahme erfolgen sollten.

\section{Retrograde Revaskularisation von Unterschenkelarterien}

Eine Revaskularisation sollte nur bei Patienten mit symptomatischer Erkrankung erfolgen. Sie sollte bei kritischer Extremitätenischämie (Rutherford-Stadien 4-6) obligatorisch angestrebt werden, wird heute aber auch bei einer den Lebensstil einschränkenden Claudicatio intermittens - unabhängig von der Gehstrecke empfohlen, wenn der therapeutische Eingriff mit hohen Erfolgschancen verbunden ist. Aufgrund der Fortschritte im endovaskulären Bereich mit Verbesserung der Interventionsmaterialien und -techniken ziehen heute viele Zentren eine Strategie des "endovascular first" für den Großteil der Revaskularisationseingriffe vor.

Die retrograde Revaskularisation von Unterschenkelarterien ist eine relativ neue Möglichkeit, die Revaskularisation zu bewerkstelligen, wenn der antegrade Zugang nicht möglich ist [33]. Der Referent hat darin viel eigene Erfahrung und erläuterte, dass diese Technik neben der Verbesserung des zur Verfügung stehenden Materials wesentlich dazu beitrug, dass die Erfolgsquote von infrapoplitealen Eingriffen in den letzten Jahren deutlich angestiegen ist.

\section{Medikamentefreisetzende, ballonexpandierbare Stents bei infrapoplitealen Läsionen}

Von den vorgestellten hochinteressanten Studien zu neuen Techniken verdienteine ganzbesondere Beachtung: Die ACHILLES-Studie mit der Untersuchung von 200 Patienten mit infrapoplitealen Stenosen zeigte, dass sirolimusfreisetzende, ballonexpandierbare Stents einer reinen Angioplastie überlegen sind. Nicht nur die Restenoserate ( $22 \%$ vs. $50 \%$ ), sondern auch die Heilungsrate von chronischen Wunden und die Lebensqualität waren darunter besser [17].

Der Referent betonte, dass gerade für kurze, proximale infrapopliteale Stenosen und Verschlüsse medikamente- 
freisetzende, ballonexpandierbare Stents eine gute Behandlungsoption darstellen.

\section{Hinweis auf das Manual des Diabetes Update 2016}

Abschließend soll hier erwähnt werden, dass in dem publizierten Manual des Diabetes Update 2016 [15] zahlreiche weitere klinische Studien referiert und kommentiert sind, die ich Ihnen wärmstens zu lesen empfehle.

Ich wünsche Ihnen viel Spaß und viele neue Erkenntnisse beim Lesen!

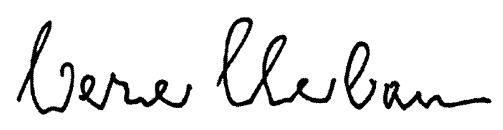

W. A. Scherbaum

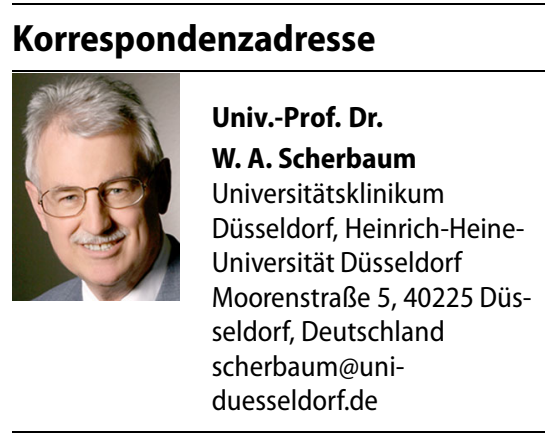

Danksagung. Den Referenten des Diabetes Update 2016 danke ich für wertvolle Diskussionen und Kommentare zu dem vorliegenden Beitrag.

Interessenkonflikt. Der Referent erklärt, dass er in den vergangenen 5 Jahren Zuwendungen für Beratertätigkeit oder Vortragshonorare von folgenden Firmen erhalten hat: Amgen, Boehringer Ingelheim, CompuGroup Medical, Lilly Deutschland, Novartis, Roche Diagnostics, Sanofi Aventis, Takeda.

\section{Literatur}

1. Althouse AD, Abbott JD, Forker AD et al (2014) Risk factors for incident peripheral arterial disease in type 2 diabetes: results from the Bypass Angioplasty Revascularization Investigation in Type 2 Diabetes (BARI 2D) Trial. Diabetes Care 37:1346-1352

2. Basaria S, Harman SM, Travison TG et al (2015) Effects of testosterone administration for 3 years on subclinical atherosclerosis progression in older men with low or low-normal testosterone levels: a randomized clinical trial. JAMA 314:570-581

3. Bateman B, Huybrechts K, Fischer M et al (2015) Chronic hypertension in pregnancy and the risk of congenital malformations: a cohort study. Am J ObstetGynecol 212(337):e1-e14

4. Boden G, Homko C, Barrero CA et al (2015) Excessive caloric intake acutely causes oxydative stress, GLUT4 carbonylation, and insulin resistance in healthy men. Sci Transl Med 7:304re7

5. Cannon CP, Blazing MA, Giugliano RP et al (2015) Ezetimibe added to statin therapy after acute coronary syndromes. N Engl J Med 372:2387-2397

6. Castillo W, Boggess K, Stürmer T et al (2015) Association of adverse pregnancy outcomes with glyburide vs Insulin in women with gestational diabetes. JAMA Pediatr 169/5:452-458

7. ChiswickC, Reynolds R, Denisan Fetal (2015) Effect of metformin on maternal and fetal outcomes in obese pregnant women (EMPOWaR): a radomised double-blind, placebo-controlled trial. Lancet Diabetes Endocrinol 2015 doi:10.1016/S2213858(15)00219-3

8. Erondu N, Desai M, Ways K (2015) Diabetic ketoacidosis and related events in the canagliflozin type 2 diabetes clinical program. Diabetes Care 38:1680-1686

9. Fedorko I, Bowen JM, Jones W et al (2016) Hyperbaric oxygen therapy does not reduce indications for amputation in patients with diabetes with nonhealing ulcers of the lowerlimb:a prospective double-blind, randomized controlled clinical trial. Diabetes Care Jan 39(3):392-399. doi:10.2337/dc15-2001

10. Fowkes FG, Rudan D, Rudan I et al (2013) Comparison of global estimates of prevalence and risk factors for peripheral artery disease in 2000 and 2010: a systematic review and analysis. Lancet 382:1329-1340

11. Fuller NR, Caterson ID, Sainsbury A et al (2015) The effect of a high-egg diet on cardiovascular risk factors in people with type 2 diabetes: the Diabetes and Egg (DIABEGG) study - a 3-month randomized controlled trial. Am J Clin Nutr 101(4):705-713

12. Garcia-Esquinas $E$, Graciani A, Guallar-Castillón $P$ et al (2015) Diabetes and risk of frailty and its potential mechanisms. A prospective cohortstudy of older adults. J Am Med Dir Assoc 16:748-754

13. Gibbons $\mathrm{CH}$, Freeman R (2015) Treatment-induced neuropathy of diabetes: an acute, iatrogenic complication of diabetes. Brain 138(Pt 1):4-52

14. Haanpää M, Cruccu G, Nurmikko TJ et al (2016) Capsaicin $8 \%$ patch versus oral pregabalin in patients with peripheral neuropathic pain. Eur J Pain 20(2):316-328

15. Hamann A, Kleinwechter H, Martin S, Stumvoll M (Hrsg) (2016) Handbuch Diabetologie 2016. med publico $\mathrm{GmbH}$, Wiesbaden

16. Jacubowicz D, Weinstein J, Ahren B et al (2015) Fasting until noon triggers increased postprandial hyperglycemia and impaired insulin response after lunch and dinner in individuals with type 2 diabetes: a randomized clinical trial. Diabetes Care 38:1820-1826

17. Katasanos K, Spiliopoulos S, Diamantopoulos A et al (2016) Wound healing outcomes and health-related quality-of-life changes in the ACHILLES trial: 1-year results from a prospective randomized controlled trial of infrapopliteal balloon angioplasty versus Sirolimus-eluting stenting in patients with ischemic peripheral arterial disease. Jacc Cardiovasc Interv 9:259-267

18. Kranke P, Bennett MH, Martyn-St JM et al (2015) Hyperbaric oxygen therapy for chronic wounds. Cochrane Database Syst Rev 6:CD004123 doi:10.1002/14651858.CD004123.pub.4

19. Lipska KJ, De Rekeneire N, Van Ness PH et al (2015) Potential overtreatment of diabetes mellitus in older adults with tight glycemic control. Jama Int Med 175(3):356-362

20. Matthaei S, Catrinoiu D, Celinski A et al (2015) Randomized, double-blind trial of triple therapy with saxagliptin add-on to dapagliflozin plus metformin in patients with type 2 diabetes. Diabetes Care 38:2018-2202

21. National Institute for Health and Care Excellence. Diabetic foot problems: prevention andmanagement. (London: Nice; 2015. (Nice Guidelines; Vol 19)) http://www.nice.org.uk/guidance/ng19

22. Pickwell K, Siermsa V, Kars Met al (2015) Predictors of lower-extremity amputation in patients with an infected diabetic foot ulcer. Diabetes Care 38:852-857

23. Raal FJ, Giugliano RP, Sabatine MS et al (2014) Reduction in lipoprotein(a) wit PCSK9 monoclonal antibody evoloculab (AMG 145): a pooled analysis of more than 1,300 patients in 4 phase II trials. J Am Coll Cardiol 63:1278-1288

24. Reinecke H, Unrath M, Freisinger E et al (2015) Peripheral arterial disease and critical limb ischaemia: still poor outcomes and lack of guideline adherence. Eur Heart J 36:932-938

25. Robinson JG, Farnier M, Krempf M et al (2015) Efficacy and safety of aliroculab in reducing lipids and cardiovascular events. N Engl J Med 372:1489-1499

26. Romagnuolo $R$, Scipione $C A$, Boffa $M B$ et al (2015) Lipoprotein(a) catabolism is regulated by proprotein convertase subtisilin/kexin type 9 through the low density lipoprotein receptor. J Biol Chem 290:11649-11662

27. Rosenstock J, Hansen L, Zee Pet al (2015) Dual addon therapy in type 2 diabetes poorly controlled with metformin monotherapy: a randomized double-blind trial of saxagliptin plus dapagliflozin addition versus single addition of saxagliptin or dapagliflozin to metformin. Diabetes Care 38:376-383

28. Sabatine MS, Giugliano PR, Wiviott SD et al (2015) Efficacy and safety of evoloculab in reducing lipids and cardiovascular events. N Engl J Med 372:1500-1509

29. Shan Z, Ma H, Xie M et al (2015) Sleep duration and risk of type 2 diabetes: a meta-analysis of prospective studies. Diabetes Care 38(3):529-537

30. Shukla AP, lliescu RG, Thomas CG et al (2015) Food order has a significant impact on postprandial glucose and insulin levels. Diabetes Care 38(7):e98-99

31. Steven S, Taylor R (2015) Restoring normoglycaemia by use of a very low calorie diet in longand short-duration type 2 diabetes. Diabet Med 32(9):1149-1155

32. Wanner CW, Inzucchi SE, Lachlin JM et al (2016) Empagliflozin and progression kidney disease in type 2 diabetes. NEJM. doi:10.1056/ NEJMoa1515920

33. Werner M, Piorkowski M, Schmidt A (2013) Technique and outcome of retrograde crural artery revascularization. J Cardiovasc Surgery 54:151-158

34. Zinman B, Wanner ZBC, Lachin JM et al (2015) Empagliflozin, cardiovascular outcomes, and mortality in type 2 diabetes (EMPA-REG). N Engl J Med 373:2117-2128 